\title{
Does higher usage of low-cost statins correlate with a poorer achievement in cholesterol quality markers for secondary prevention?
}

James Hickman

\begin{abstract}
There is pressure on primary care trusts, and therefore on GPs, to reach specific levels of use of low-cost statins as a proportion of total statin prescribing. This simple study looks at some markers of the quality of the results achieved. A correlation is found between a higher proportion of low-cost statin prescribing and lower achievement raising questions as to whether financial savings may be offset by poorer results. Keywords

cholesterol; hydroxymethylglutaryl-CoA reductase inhibitors; Quality and Outcomes Framework; quality indicators; secondary prevention; statins.
\end{abstract}

J Hickman, DRCOG, DFFP, FIMC RCS(Ed), FRCGP, GP, North Curry Health Centre, Taunton, Somerset.

Address for correspondence James Hickman, North Curry Health Centre, Greenway, North Curry, Taunton TA3 6NQ.

E-mail: james.hickman@northcurryhc.nhs.uk

Submitted: 27 February 2009; Editor's response: 17 April 2009; final acceptance: 1 June 2009.

(C)British Journal of General Practice 2010; 60: 50-52.

DOI: 10.3399/bjgp10X482112

\section{INTRODUCTION}

The National Institute for Health and Clinical Excellence (NICE) has recommended that statin (hydroxymethyl glutaryl [HMG]-CoA reductase inhibitor) therapy should usually be initiated with a drug with a low acquisition cost. ${ }^{1}$ The NHS Institute has taken this a step further in developing an 'indicator' for primary care trusts (PCTs). In its guidance NHS Better Care, Better Value, it defines a clinical productivity indicator, 'increasing lower cost statin prescribing' as a percentage of total statin prescribing that is for low cost statins (simvastatin and prevastatin). ${ }^{2}$ At the time of writing, it stated 'if PCTs with below $77 \%$ (achieved by the top quartile of trusts) of lower cost statins increased this to $77 \%$ over £24 million would be saved in a year'.

Although the NHS Institute specifies that 'these indicators are not targets', PCTs have encouraged practices to switch patients to lower-cost statins. In Somerset the percentage of low-cost statins prescribed has been explicitly set as one of the prescribing targets for practices engaged in the practice-based commissioning enhanced service, with an expectation that $80 \%$ of all statin prescriptions should be for simvastatin or pravastatin, these being two statins with the lowest 'acquisition costs' in the UK. ${ }^{3}$

The lower-cost statins tend to be less powerful in lowering cholesterol levels. ${ }^{4}$ This simple study was therefore designed to assess whether there is a correlation between percentage use of low-cost statins and performance in managing cholesterol.

The Quality and Outcomes Framework (QOF), established as part of the national GP contract in the UK in 2004, analyses patient records to assess performance against a variety of clinical indicators. Two of these specifically look at management of cholesterol for secondary prevention.

'CHD8' counts the percentage of the practice patient population, with a history of ischaemic heart disease, whose most recent total cholesterol was below $5.0 \mathrm{mmol} / \mathrm{l}$. 
'Stroke8' counts the percentage of the practice patient population, with a history of cerebrovascular disease, whose most recent total cholesterol was below $5.0 \mathrm{mmol} / \mathrm{l}$.

The study performed a simple analysis to compare the 'performance' of Somerset practices in achieving a high percentage of low-cost statin prescribing against their performance in these two QOF markers.

\section{METHOD}

Somerset PCT regularly sends to all their practices a 'prescribing scorecard' which details practices' performance against various prescribing indicators. One of these indicators is the amount of simvastatin and pravastatin prescribed as a percentage of total statin prescribing.

The website www.gpcontract.co.uk provides a detailed breakdown of performance of individual practices against each QOF marker. This includes the percentage achievement results achieved for CHD8 and Stroke8.

The statin prescribing data from the prescribing scorecard for April 2007 were compared with the performance in managing cholesterol as measured by the data for the QOF for the financial year 2006-2007.

\section{RESULTS}

Practice use of the 'low acquisition cost' statins, simvastatin and pravastatin, ranged from $33.6 \%$ to $93.6 \%$, with a median of $72.0 \%$. Practice achievement of CHD8 ranged from $36.4 \%$ to $98.1 \%$, with a median of $82.7 \%$. Practice achievement of Stroke8 ranged from $46.7 \%$ to $97.2 \%$, with a median of $75.7 \%$.

Figure 1 is a scatter graph showing the practices'

\section{How this fits in}

Statins have been demonstrated to be effective for secondary prevention of cardiovascular events and stroke. This study raises the concern that targets for practices to achieve high use of low-cost statins may be related to poorer achievement of cholesterol quality markers. There is a potential tension between the expectation to use cheaper medications and the drive to reach clinical targets.

performance on the statin prescribing scorecard plotted against achievement in cholesterol management in patients with coronary heart disease and cerebrovascular disease.

There is one (very) outlier practice with very low achievement on coronary heart disease and stroke. Given the nature of the rest of the data and that this is the only obvious outlier, it was felt reasonable to omit this outlier and consider parametric (Pearson's) correlations. Using these methods, the correlation between statin choice and CHD8 achievement is $-0.26, P=0.028$. Coincidentally, exactly the same correlation is found for Stroke8 achievement. The correlation is statistically significant by usual standards of $<0.05$ and therefore unlikely to have arisen by chance.

A key potential confounding factor regarding these data is the practice of exception reporting. Under the QOF rules, patients, who would otherwise be expected to fall within the denominator population, can be 'exception reported' for reasons such as intolerance of medication or being deemed as 'unsuitable' by their GP. The study therefore went on to use exception reporting data for the practices, obtained from the NHS Information Centre, to test whether there was a correlation with low- or high-

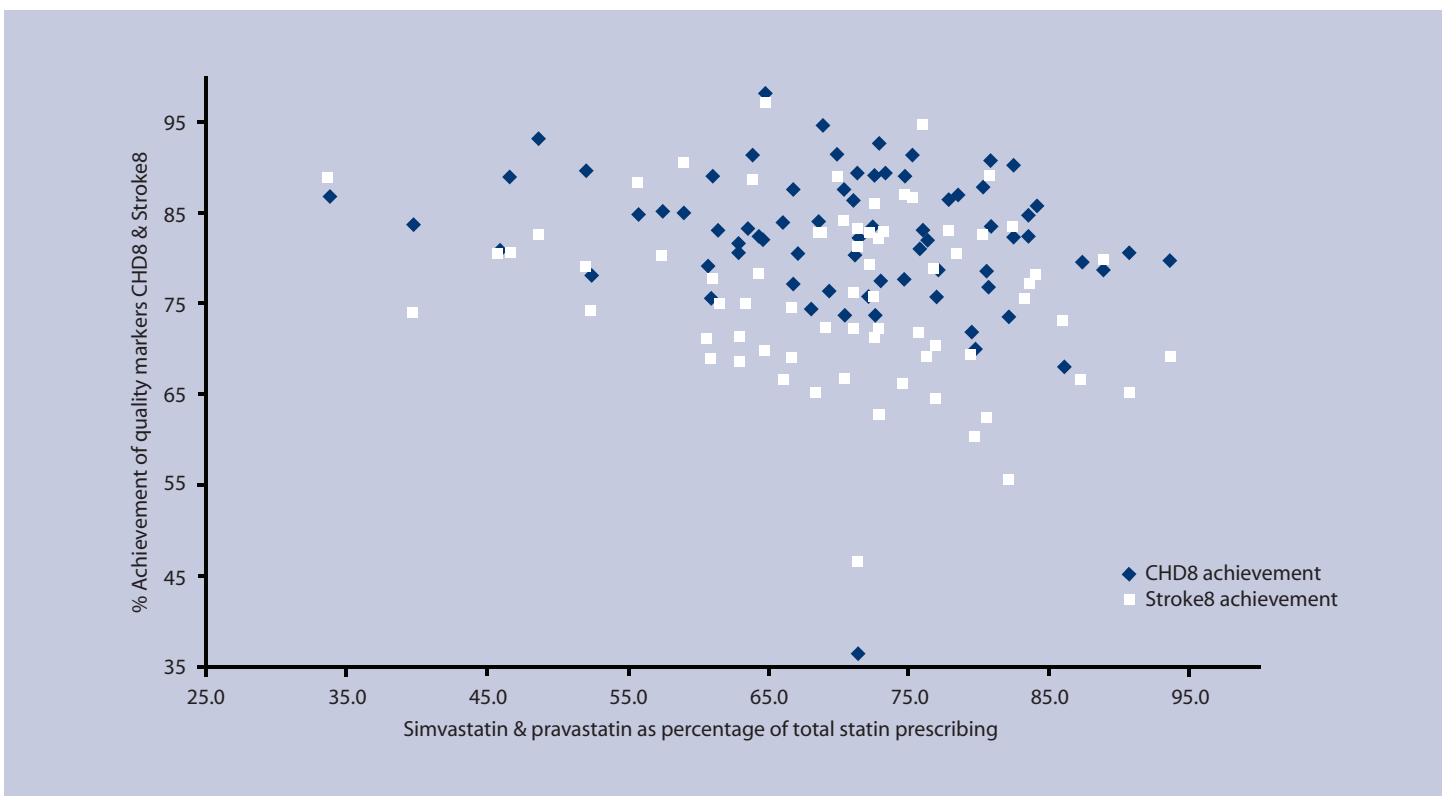

Figure 1. Achievement of cholesterol quality markers versus use of low-cost statins. 


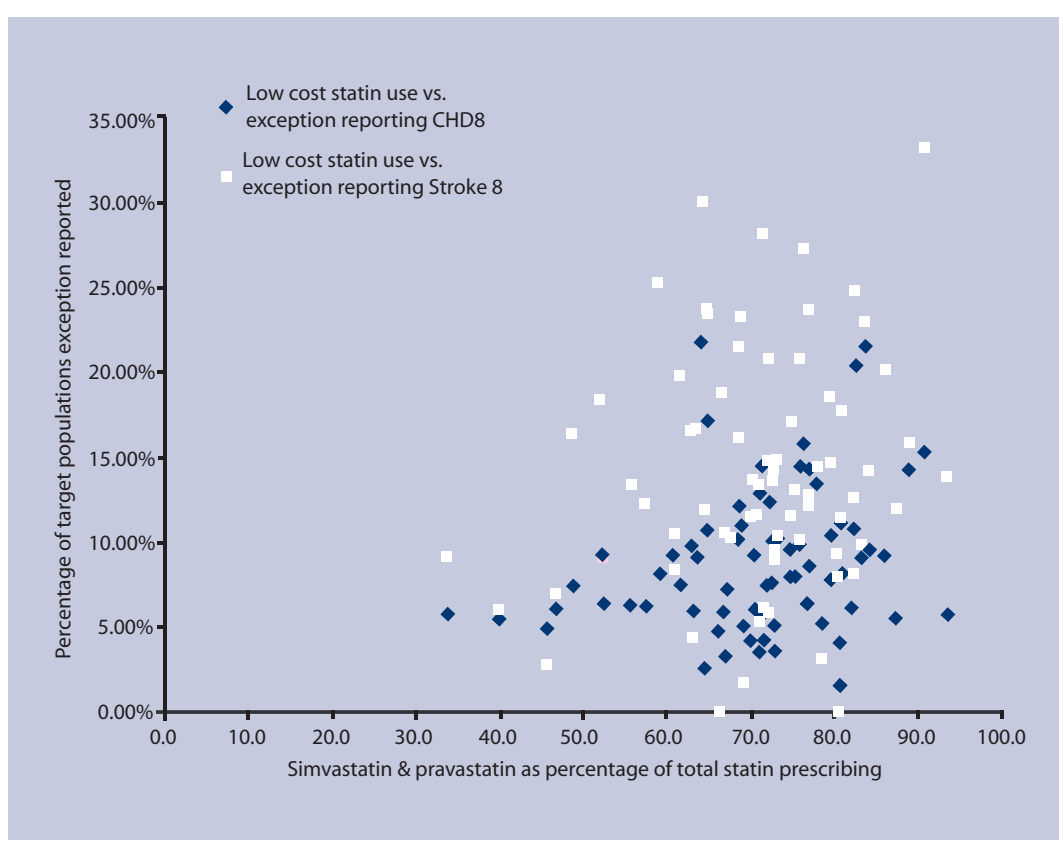

Figure 2. Cholesterol quality marker exception reporting versus use of low-cost statins. cost statin prescribing. The data are presented in Figure 2.

\section{DISCUSSION}

There does appear to be a correlation between an increasing proportion of 'low-cost' statin use and decreasing practice performance against the QOF indicators for secondary prevention.

In the light of the initial results, the study had considered whether practices with greater usage of more costly statins might also tend to have higher levels of exception reporting, making results easier to achieve. In fact the data, as shown in Figure 2, suggest that the converse is true with practices with greater use of 'low acquisition cost' statins tending to resort to higher exception reporting.

As the 'low acquisition cost' statins are defined as being simvastatin and pravastatin, the study also considered whether the variation in performance could be affected by which of these two statins practices tended to use. However, a review of data of relative simvastatin and pravastatin prescribing would appear to make this unlikely. Somerset as a whole has a very low prescribing rate of pravastatin. The number of items of pravastatin prescribed across Somerset for the year in question was only $2.66 \%$ of the total 'low-cost' statin prescriptions. For individual practices, the figure ranged from $0 \%$ to $23.95 \%$ but the median was $1.81 \%$, with only three practices prescribing more than $10 \%$.

There are other potential confounding factors that may independently affect a practice's achievement against QOF markers. Issues such as cardiovascular disease prevalence and deprivation may also affect a practice's statin use and performance, but this in itself may be an argument against applying blanket prescribing targets without considering such factors.

It would be of interest to look at those outliers who achieve good results with high proportional use of low-cost statins. It may be that some are making more use of additional therapy such as ezetimibe (as recommended by NICE) to augment treatment. ${ }^{5}$ With the cost of 28 days of ezetimibe being more than that of $20 \mathrm{mg}$ daily of either atorvastatin or rosuvastatin, this may counteract the savings achieved.

The QOF markers are less stringent than the targets set by NICE in its most recent guidance, which recommends a maximum cholesterol target of $4 \mathrm{mmol} / \mathrm{l}$ for secondary prevention. ${ }^{6}$ It is possible that the achievement graphs might be steeper if the data were available to compare performance against these standards.

The results raise the concern that the pressure to prescribe low-cost statins may contribute to poorer control of cholesterol at the practice population level. Whether this has significant effects on mortality and morbidity is beyond the scope of this simple study, but NICE recommendations and QOF markers are based on the premise that tight control of cholesterol has significant benefits for secondary prevention. This de facto target for use of 'low acquisition cost' statins seems crude and does not allow for the characteristics of differing practice populations.

\section{Ethics committee}

\section{Not applicable}

\section{Competing interests}

The author is a Somerset dispensing GP whose practice has a $48.6 \%$ 'low acquisition cost' statin prescribing rate as at March 2009

\section{Acknowledgements}

I would like to thank Dr Paul Ewings, Head of Research and Development at Taunton and Somerset NHS Trust for his advice and help with analysis of the data.

\section{Discuss this article}

Contribute and read comments about this article on the Discussion Forum: http://www.rcgp.org.uk/bjgp-discuss

\section{REFERENCES}

1. National Institute for Health and Clinical Excellence. NICE Technology Appraisal Ta94. Cardiovascular disease - statins. London: NICE, 2006. http://www.nice.org.uk/nicemedia/pdf/TA094quickrefguide.pdf (accessed 28 Oct 2009).

2. NHS Institute for Innovation and Improvement. NHS indicators. http://www.productivity.nhs.uk/Definitions.aspx (accessed 2 Nov 2009).

3. Somerset Primary Care Trust. Specification for a local enhanced service 3.2. practice based commissioning. Yeovil: Somerset Primary Care Trust, 2007.

4. Jones PH, Davidson MH, Stein EA, et al. Comparison of the efficacy and safety of rosuvastatin versus atorvastatin, simvastatin, and pravastatin across doses (STELLAR ${ }^{\star}$ Trial). Am J Cardiol 2003; 92(2): $152-160$.

5. National Institute for Health and Clinical Excellence. NICE Technology Appraisal Ta132. Hypercholesterolaemia - ezetimibe. London: NICE, 2007. http://guidance.nice.org.uk/TA132 (accessed 2 Nov).

6. National Institute for Health and Clinical Excellence. NICE Guideline CG67. Lipid modification. London: NICE, 2008. http://guidance.nice.org.uk/TA132 (accessed 2 Nov). 